\section{INFLAMMATORY ACTIONS, AND THEIR} TREATMENT.

By C. Handfiemd Jones, M.B., F.R.S., Assistant-Physician to St. Mary's Hospital.

[Concluded from page 233.]

Quitring now the remedies for sthenic inflammation, let us turn to those which are appropriate to asthenic. The contrast between the two groups is full of instruction.

The intluence of nitric acid in toning the vessels of the bronchial membrane when relaxed, as in asthenic catarrhal affections is very clear and positive. Râles with cough and expectoration that will not yield to antimony or ipecacuan, or which return when these medicines have been too long continued, disappear under its use. We cannot imagine it to act otherwise than by an impression on the nerves: taken into the blood, it must combine with a base, soda probably, and its action as a salt would surely be very different from that which we have just noticed. Its nervine influence is further attested by its remarkable efficacy in arresting the paroxysms of pertussis, as has been well shown by Dr. Gibbs.

That quinine, strychnine, and arsenic, are nerve toners, cannot, I think, be any matter of question. If malarious poison acts paralysingly and depressingly on the whole nervous system, if the fevers and congestions which it produces can be explained by our hnowledge of the laws of action of the sympathetic nerves, then surely quinine, and in a less degree arsenic, which counteracts so powerfully this wide spread poison, must act also on the same system. It is matter of everyday experience that asthenic inflammatory processes, and fluxes proceeding from them, can be checked and arrested by the three above-named agents. The change effected by the skilful administration of arsenic in cases of skin disease appropriate for its use, is very striking and instructive. The abnormally increased heat, the hyperamia, the serous exudation (taking a case of eczema or pempligus), cease, and except some lingering congestion of the capillaries, the part returns to its healthy state. How very like this is to the effect of galvanising the upper end of the divided sympathetic nerve in the neck of an animal, as described by Bernard! It is clear that arsenic does not cure a skin eruption in virtue of some special relation it has for cutaneous tissue, because it will act very similarly on the intestinal mucous membrane, checking or arresting chronic diarrhcea or dysentery; as also on the uterus, restraining menorrhagia and leucorrhoa, according to Mr. Hunt and $\mathrm{Dr}$. Locock. The great point necessary to be borne in mind in the use of these and all nerve tonics, is that they act also, or are liable to act, as tissue irritants; so that it may require much care and management to obtain the first action without the second. If, therefore, there exist any amount of sthenic in flammation (a point not by any means always easy to determine), these agents are quite inappropriate. Carbonate of am monia seems to be the remedy of this class which has the least tendency to cause irritation of tissuo, At least this is true in the case of the pulmonary organs.

Quinine and arsenic evince particularly their power as nerve tonics from their known good effects in all neuralgiæ; they seem to operate more on the sensory than on the motor nerves. The reverse is the case with strychnine; it serves us in paralysis of voluntary muscles, not depending on a central lesion, and in torpor of the intestinal muscular coats, as also, according to Dr. Vernon, in uterine inertia. I will presently allude to several other drugs which possess a like nerve toning influence moro or less decidedly, but those now mentioned are the best exemplars.

A consideration of their action, and of their relation to the nervous system, strongly confirms the opinion that typical asthenic inflammation is chiefly dependent on palsy or weakening of the vaso-motor nerves of the part. This does not, of course, exclude the influence of a poison received into the blood as in some cases the cause of the paralysis; but while fully admitting this, it brings to our minds the very important fact, that the degree of power and tone of the nervous system very materially determines whether the roison produces its effects or not. On this it seems impossible to lay too much stress. How striking is the fact, that eight grains of quinine daily will enable a man to underco exposure with impunity to the malaria of the west coant of Africa, which acts with such deadly effect on the unprotected. Yet, of necessity, the same poison must enter by respiration into the circulation of each. If two persons are exposed to the miasm of typhoid or typhus fever, there is considerably more likelihood of the one who is weakly and depressed being affected by it than the other who is in full tone and vigour. Yet both inhale the poison into their blood alike; only the one succumbs to it, the other resists it. In the case of malarious disorder, where the system has often suffered and retains the morbid impregnation, so that a slight cause reproduces the phenomena, what else avails to keep them in abeyance but raising and maintaining the tone and vigour of the system? The poison is there always, but it makes all the difference whether the resisting power be weak or strong. In the case of syphilitic disease, as already remarked, we find the same law holds true. Quite to the same effect are the instances of disease affecting a defeated army far more than a victorious one, and of the remarkable influence of ennui and disappointment in engendering disease, as of excitement and exhilaration in preventing it. (Vide p. 120, 121, of Mr. Martin's work.) Surely the conclusion from all this must be, that in the great majority of cases of disease, we are much rather to think of stilling perverted and excited action, and of imparting healthy tone where this is wanting, than of eliminating a poison from the system. According to the prevailing theory, we ought to cure an eczema, with its serous flux, by eliminating the (supposed) morbid element from the blood. In practice, we do cure it by toning the relaxed vessels; and well satisfied are we, as well as our patient, when we have dore so. With a bronchial, gastric, intestinal, uterine flux, we deal in a like way; and there seems no reason to question the propriety of the practice. Certainly an eliminant treatment would have a very different result. Yet in many of these cases the existence of some materies morbi might be reasonably supposed. Influenza surely depends on the action of a poison on the system, yet it would not be very wise to treat a case by attempting to eliminate the morbid agent. The plan of treating cholera by castor oil professes to be based on the view of eliminating the morbid poison. Believ ing that it may be useful in certain cases, I think the mode of its operation is altogether different. It probably sets up a different kind of action in the gastro-intestinal mucous surface. which supersedes that of cholera. This would be analogous to. the treatment of purulent ophthalmia by nitrate of silver in substance applied to the inflamed conjunctiva, or that of gonor rhœa by injection of a strong solution of the same agent (gr. x ad $\xi i)$. The remedial action in these instances is by no means. that of a mere astringent; it modifies the vitality of the affected tissue, to borrow an expression of Dr. Bennett's.

The following remarks respecting the action of some of our ordinary remedies may properly conclude this paper.

Opium may be regarded as a tissue sedative in general, not one of the nerve tissue only. As it "locks up the secretions" by staying the active function of their gland-cells, so it calms. the excited state of an inflamed membrane, e.g., in peritonitis, and stops the morbid attraction of blood thither. To do this effectually, however, it is essential that the force of the heart's contraction should be diminished by venesection, or otherwise. In an experiment I performed, thirty-three drops of liquor opii sedativus being taken in twenty-four hours, the result was that the whole quantity of urine was increased by four ounces, the acidity raised from 65 to 85 , the urea diminished by 25.514 grains, and the uric acid by 4.6 grains. The specific gravity fell from 1028 to 1020 . The stools were clayey. Opium thus evidently checks waste, and does so by calming an over-excited state of the nervous and other tissues. Under its influence fatigue of body or mind can be better endured, because the tissues have more stability, and disintegrate less readily.

Belladonna, unquestionably a nerve sedative, may perhaps. be regarded as a sedative to other tissues also. In its dilating the iris, and diminishing the contraction of other spthineters, in its calming an excited action of the heart, and arresting the secretion of the mammary gland, we have instances of its power as a local application, which are scarcely to be referred to its influence upon the nerves. (Ludwig's opinion, quoted by Dr. Harley in the Edinburgh Monthly Medical Journal, February 1857.)

Digitalis appears to act as a tonic or stimulant to vaso-motor nerves. It stills the action of the heart, probably by a stimulating operation on the pneumogastric, or the medulla oblongata, as in Weber's experiment of passing a galvanic current through the latter centre. If this be carried too far, the heart's action is arrested altogether. Like other nerve tonics, it acts best in states of debility, and " seldom succeeds in men of great natural strength, of tense fibre, of warm skin, of florid complexion, or those with a tight and cordy pulse." It also acts as a vessel toner in the arrest of menorrhagia and 
some other hæmorrhages. Its diuretic action must be referred to a stimulant operation upon the kidney, in which it resembles the following.

Oleum Terebinthine is known to be effectual in gastric and renal hæmorrhage. It has been found very useful in checking the black vomit of yellow fever. I have seen it arrest the hæmorrhagic renal flux, called chylous urine, after gallic acid and tincture of sesquichloride of iron had failed. It is of efficacy also in asthenic iritis, and in some bronchial fluxes. Its operation on the nervous system is attested by the unquestionable benefit which has resulted from its use in sciatica and other neuralgias, in chronic rheumatism, and in epilepsy. (Watson's Practice of Medicine, vol. i, p. 6:36.) These I do not view as isolated facts, but consider oil of turpentine as a nervine stimulant, operating both on the cerebro-spinal and sympathetic systems. Like many other remedies of the same class, it is a tissue-irritant; and it is necessary to manage well to obtain the first action without the second.

Balsam of Copaiba may be mentioned as a remedy of very similar nature. In vesical catarrh "it robs the urine of its mucus," and in gastric hæmorrhage it has been found to act as an efficient styptic; Dr. Churchill has derived benefit from it in cases of uterine catarrh. I would say once for all that I should never expect from this or any other nervine remedy constant results. It seems impossible to ascertain accurately the conditions of success; in the greater number of cases by far the nerves we wish to influence are quite removed from all observation; and if we had them directly under our eyes we have no means of estimating the state of the nerve force, or the special idiosyncrasy of the individual. It may be easy to see what kind of remedy is wanted in a given case, but it may be very difficult to find that which is exactly appropriate.

Ergot seems to possess a decided influence over organic muscular fibre. It powerfully contracts the walls of the developed uterus; it is recorded to have arrested epistaxes and other hæmorrhages ( J)r. Walshe praises it in epistaxis); leucorrhoal discharges are controlled by it; and when taken for a length of time, as in the case of peasants feeding on diseased rye, it causes such contraction of the arteries that the limbs pass into the state of dry gangrene. This influence may certainly be a direct one upon the muscular tissue, to which it is conveyed by the blood; it is, however, worth observing that ergot does produce marked effects on the nervous system. When taken in the food in quantity it very commonly causes a kind of intoxication, said not to be disagreeable; and as a remedial agent it is reported to have cured paraplegia and paralysis of the bladder.

Iron is ranked by 'Trousseau (and justly) as an analeptic, like oleum morrhure, ministering to the repair of blood and tissue. But it is also eminently a nerve tonic, a powerful means of combating neuralgia and neuroses of the cerebro-spinal system, as well as of restoring the power of the sympathetic. It is needless to cite any instances of its beneficial action on the cerebro-spinal nerves; but some of its infiuence on the sympathetic are necessary for my purpose. Liquor ferri pernitratis is highly spoken of by Dr. Graves as of much avail in chronic diarrhoa. 'Tincture of sesquichloride of iron has proved very beneficial in cases of erysipelas, and of bloody and albuminous urine comnected with scarlatina. Cruveilhier regards iron as a specific for engorged conditions of the spleen; and the experience of Indian physicians is also in favour of its decided efficacy. Renal hæmorrhage and vesical have been arrested by the use of tincture of sesquichloride of iron. Pre. parations of iron are found to check bronchial fluxes, uterine leucorrhoca, and passive menorrhagia. These instances show the influence of iron over the vascular system; and when they are viewed with reference to its positive effects on the cerebrospinal nerves, scarce any doubt can exist that it is by its energising the sympathetic plexuses that the contractility of the arterial walls is again brought into play.

The modus operandi of astringents requires some notice, as they approach in their effects very nearly to the tonics. It cannot, I think, be affirmed so decidedly of them that they act through the nervous system; yet neither can I agree with those who regard them as affecting living in the same way almost as dead tissue. I will briefly refer to the known effects of some of this class.

Sulphuric acid is of positive efficacy in the following conditions. It checks profuse sweats; it relieves itching and tingling of the skin in papular eruptions; it arrests profuse serous diarhoc. In the two first of these cases it surely does not act by a mere physical influence, and in the latter it seems quite open to suppose the same. Considering its relation to nitric acid (so unquestionably a nerve tonic), and its irritant local action, $I$ incline to think it must also be ranked as a nerve tonic.

Alum, besides acting as a local astringent, has a positive efficacy in lead colic, and in various hemorrhages and fluxes. Its action in colic, and intestinal neuralgia, approximates it certainly to iron and other nerve tonics.

Acetate of lead is well known as of efficacy in various hæmorrhages and fluxes, the bronchial perhaps more especially. The positive irritant influence of lead on the abdominal nerves in colic inclines me to think that it is by toning the vaso-motor that it checks hamorrhagic and other exudations. It would not form a valial objection to this view that its more prolonged operation was palsying to cortain cereloro-spinal nerves; in fact, even on the brain it sometimes acts as an irritant, producing convulsions. The great recommendation of lead seems to be, that while it tones vaso-motor nerves, it exerts a sedative action on inflamed tissnes, and might well have been noticel among that group of remedies. In this it differs from all the stronger nerve tonics.

Tannin, and Gallic acid. Although Dr. Bayes states that an overdose of the latter causes the pulse to become small and wiry, the face to grow pale, and a whizzing sound in the ears (symptoms which appear to indicate an affection of the brain and vaso-motor nerves), yet, as $I$ am not aware of any positive proof that the above agents act on the nerrous system in any special and direct manner, I do not include them in the list of nerve tonics. Their action is probably on the homogeneous wall of the capillaries, rendering it less permeable to the liquor sanguinis. Simple and structureless as this wall appears on the most careful examination, it must yet be possessed of some remarkable qualities. In most situations it allows (in health) a certain amount of albuminous plasma to transule its texture, just what is needed for the nutrition of the muscle or other tissue to which it belongs. In the Malpighian capsules it allows a large quantity of watery fluid to drain off absolutely deprived of albumen, but probably containing some saline matter. In the air-cells of the lungs it merely allows a little moisture to exude, which is soon converted into vapour. This peculiar filtering power is, however, easily disturbed; an increased strain upon the delicate structure causes the effusion of albuminous aud fibrinous fluid, it may be in large quantity. The escape of blood globules in greater or less amount is usually thought to imply rupture, and it seems scarce possible it should be otherwise. Yet perhaps the established fact of copious hæmorrhage occurring without detectable breach of surface may justify us in concluding that the capillary wall must be capable of undergoing such alteration in its composition as to allow shaped corpuscles to pass through it. There is a good deal of resemblance between the capillary membrano and fibrinous films; and it is very much in states where fibrine is deficient or imperfectly formed that hamorrhages are apt to take place. In such conditions, I think it by no ineans impro bable that the homogeneous capillary membrane loses its duo consistence, and becomes semi-liquid, like soft jelly ; so that, without actually tearing, it yet opposes small let or hindrance to the escape of the blool within the tube. Less degrees of the same condition would probably allow the escape of albuminous and fibrinous fluid. To such, which are almost always asso ciated with deficient contractility of the arterial coats, I should think tannin and gallic acid especially appropriate. It is, moreover, easily comprehensible on this view how these remedies should often fail in arresting hamorrhage; for if the smoll arteries are gaping wide, and flushing the part copiously with blood, it would be impossible for the weak walls of the capillaries to gather strength to withstand the strain. It is, therefore, higlily rational practice to conjoin strychnia or quinime with gallic or tannic acid, so as to accomplish both objects. On the other hand it might be uscless to tone the small arteries, if the capillaries remained very lax and weak.

In the foregoing observations I hare merely attempted to gather up the general bearing of a number of well-known facts, without advancing anything new. Evidence of this kind, though less pretending to exactness than the determinations of the microscope and balance, has yet a value of its own, and is more available for the exigencies of practice. I will only add one remark in conclusion. Our remedies act on the vital qualities of the tissues; and if these are greatly enfeebled and depressed but little is to be expected from them. Rest, fresh air, good food, are the best restorers of natural endowments; after these if need be, let drugs be emplosed. Is this as much thought of as it should be? 